\title{
End-Completely-Regular and End-Inverse Lexicographic Products of Graphs
}

\author{
Hailong Hou and Rui Gu \\ School of Mathematics and Statistics, Henan University of Science and Technology, Luoyang, Henan 471003, China \\ Correspondence should be addressed to Hailong Hou; hailonghou@163.com
}

Received 20 February 2014; Accepted 6 April 2014; Published 17 April 2014

Academic Editor: Wu Tongsuo

Copyright ( $) 2014$ H. Hou and R. Gu. This is an open access article distributed under the Creative Commons Attribution License, which permits unrestricted use, distribution, and reproduction in any medium, provided the original work is properly cited.

\begin{abstract}
A graph $X$ is said to be End-completely-regular (resp., End-inverse) if its endomorphism monoid End $(X)$ is completely regular (resp., inverse). In this paper, we will show that if $X[Y]$ is End-completely-regular (resp., End-inverse), then both $X$ and $Y$ are Endcompletely-regular (resp., End-inverse). We give several approaches to construct new End-completely-regular graphs by means of the lexicographic products of two graphs with certain conditions. In particular, we determine the End-completely-regular and End-inverse lexicographic products of bipartite graphs.
\end{abstract}

\section{Introduction and Preliminary Concepts}

Endomorphism monoids of graphs are generalizations of automorphism groups of graphs. In recent years, much attention has been paid to endomorphism monoids of graphs and many interesting results concerning graphs and their endomorphism monoids have been obtained. The aim of this research is to develop further relationship between graph theory and algebraic theory of semigroups and to apply the theory of semigroups to graph theory. The bipartite graph is a class of famous graphs. Their endomorphism monoids are studied by several authors. In [1], the connected bipartite graphs whose endomorphism monoids are regular were explicitly found. In [2], Fan gave a characterization of connected bipartite graphs with an orthodox monoid. The joins of bipartite graphs with regular endomorphism monoids were characterized in [3]. The joins of bipartite graphs with completely regular endomorphism monoids were characterized in [4]. The endomorphism monoids and endomorphism regularity of graphs were considered by several authors (see [5-8]). In this paper, we will characterize the End-completely-regular and End-inverse lexicographic products of two graphs. We give several approaches to construct new End-completely-regular graphs by means of the lexicographic products of two graphs with certain conditions. In particular, we will determine the End-completely-regular and End-inverse lexicographic products of bipartite graphs.
The graphs $X$ considered in this paper are undirected finite simple graphs. The vertex set of $X$ is denoted by $V(X)$ and the edge set of $X$ is denoted by $E(X)$. If two vertices $x_{1}$ and $x_{2}$ are adjacent in $X$, the edge connecting $x_{1}$ and $x_{2}$ is denoted by $\left\{x_{1}, x_{2}\right\}$ and we write $\left\{x_{1}, x_{2}\right\} \in E(X)$. A subgraph $H$ is called an induced subgraph of $X$ if for any $a, b \in V(H)$, $\{a, b\} \in E(H)$ if and only if $\{a, b\} \in E(X)$. A graph $X$ is called bipartite if $X$ has no odd cycle. It is known that if a graph $X$ is a bipartite graph, then its vertex set can be partitioned into two disjoint nonempty subsets such that no edge joins two vertices in the same set.

Let $X$ and $Y$ be two graphs. The join of $X$ and $Y$, denoted by $X+Y$, is a graph such that $V(X+Y)=V(X) \cup V(Y)$ and $E(X+Y)=E(X) \cup E(Y) \cup\left\{\left\{x_{1}, x_{2}\right\} \mid x_{1} \in V(X), x_{2} \in V(Y)\right\}$. The lexicographic product of $X$ and $Y$, denoted by $X[Y]$, is a graph with vertex set $V(X[Y])=V(X) \times V(Y)$, and with edge set $E(X[Y])=\left\{\left\{(x, y),\left(x_{1}, y_{1}\right)\right\} \mid\left\{x, x_{1}\right\} \in E(X)\right.$, or $x=x_{1}$ and $\left.\left\{y, y_{1}\right\} \in E(Y)\right\}$. Denote $Y_{x}=\{(x, y) \mid y \in V(Y)\}$ for any $x \in V(X)$.

Let $X$ and $Y$ be graphs. A mapping $f$ from $V(X)$ to $V(Y)$ is called a homomorphism (from $X$ to $Y$ ) if $\left\{x_{1}, x_{2}\right\} \in E(X)$ implies that $\left\{f\left(x_{1}\right), f\left(x_{2}\right)\right\} \in E(Y)$. A homomorphism $f$ is called an isomorphism if $f$ is bijective and $f^{-1}$ is a homomorphism. A homomorphism (resp., isomorphism) $f$ from $X$ to itself is called an endomorphism (resp., automorphism) of $X$ (see [9]). The sets of all endomorphisms and automorphisms 
of $X$ are denoted by $\operatorname{End}(X)$ and $\operatorname{Aut}(X)$, respectively. A graph $X$ is said to be unretractive if $\operatorname{End}(X)=\operatorname{Aut}(X)$. For any $f \in \operatorname{End}(X)$, it is easy to see that $f \in \operatorname{Aut}(X)$ if and only if $f$ is injective.

A retraction of a graph $X$ is a homomorphism $f$ from $X$ to a subgraph $Y$ of $X$ such that the restriction $\left.f\right|_{Y}$ of $f$ to $V(Y)$ is the identity mapping on $V(Y)$. In this case, $Y$ is called a retract of $X$. It is known that the idempotents of $\operatorname{End}(X)$ are retractions of $X$. Denote by $\operatorname{Idpt}(X)$ the set of all idempotents of $\operatorname{End}(X)$. Let $f$ be an endomorphism of a graph $X$. A subgraph of $X$ is called the endomorphic image of $X$ under $f$, denoted by $I_{f}$, if $V\left(I_{f}\right)=f(V(X))$ and $\{f(a), f(b)\} \in E\left(I_{f}\right)$ if and only if there exist $c \in f^{-1}(f(a))$ and $d \in f^{-1}(f(b))$ such that $\{c, d\} \in E(X)$. By $\rho_{f}$ we denote the equivalence relation on $V(X)$ induced by $f$; that is, for $a, b \in V(X),(a, b) \in \rho_{f}$ if and only if $f(a)=f(b)$. Denote by $[a]_{\rho_{f}}$ the equivalence class containing $a \in V(X)$ with respect to $\rho_{f}$.

An element $a$ of a semigroup $S$ is called regular if there exists $x \in S$ such that axa $=a$. An element $a$ of a semigroup $S$ is called completely regular if $a=a x a$ and $x a=a x$ hold for some $x \in S$. A semigroup $S$ is called regular (resp., completely regular) if all its elements are regular (resp., completely regular). An inverse semigroup is a regular semigroup in which the idempotents commute. A graph $X$ is said to be End-regular (resp., End-completelyregular, End-inverse) if its endomorphism monoid $\operatorname{End}(X)$ is regular (resp., completely regular, inverse). Clearly, Endcompletely-regular graphs as well as End-inverse graphs are End-regular.

For undefined notation and terminology in this paper, the reader is referred to [9-14]. We list some known results which will be used in the sequel.

Lemma 1 (see [2]). If $X[Y]$ is End-regular, then both $X$ and $Y$ are End-regular.

Lemma 2 (see [15]). Let $G$ be a graph and let $f \in \operatorname{End}(G)$. Then $f$ is completely regular if and only if $\left.f\right|_{I_{f}} \in A u t\left(I_{f}\right)$.

Lemma 3 (see [15]). Let $X$ be a bipartite graph. Then $X$ is Endcompletely-regular if and only if $X$ is one of $K_{1}, K_{2}, P_{2}, 2 K_{1}$, $2 K_{2}$, and $K_{1} \cup K_{2}$.

Lemma 4 (see [4]). Let $X$ and $Y$ be two bipartite graphs. Then $X+Y$ is End-completely-regular if and only if one of them is End-completely-regular and the other is $K_{1}$ or $K_{2}$.

Lemma 5 (see [16]). Let $G$ be a graph and $f \in \operatorname{End}(G)$. Then $f$ is completely regular if and only if there exists $g \in \operatorname{Idpt}(G)$ such that $\rho_{g}=\rho_{f}$ and $I_{g}=I_{f}$.

Lemma 6 (see [4]). Let $X$ be a bipartite graph. Then $X$ is Endinverse if and only if $X=K_{1}$ or $X=K_{2}$.

Lemma 7 (see [17]). Let $X$ and $Y$ be two graphs. Then $\operatorname{End}(X[Y])=\operatorname{End}(X)[\operatorname{End}(Y)]$ if and only if for any $f \in$ End $(X[Y])$ and $x \in V(X)$, there exists $x^{\prime} \in V(X)$ such that $f\left(Y_{x}\right) \subseteq Y_{x^{\prime}}$.

\section{Main Results}

In this section, we will characterize the End-completelyregular and End-inverse lexicographic products of two graphs. We first show that if $X[Y]$ is End-completely-regular, then both $X$ and $Y$ are End-completely-regular.

Theorem 8. Let $X$ and $Y$ be two graphs. If $X[Y]$ is Endcompletely-regular, then both $X$ and $Y$ are End-completelyregular.

Proof. By Lemma 2, to show that $X$ is End-completelyregular, it is only necessary to verify that $\left.f\right|_{I_{f}}$ is an automorphism of $I_{f}$ for each $f \in \operatorname{End}(X)$. Define a mapping $F$ from $V(X[Y])$ to itself by

$$
F((x, y))=(f(x), y) \quad \forall(x, y) \in V(X[Y]) .
$$

Then $F \in \operatorname{End}(X[Y])$. Since $X[Y]$ is End-completely-regular, by Lemma $2,\left.F\right|_{I_{F}}$ is an automorphism of $I_{F}$. It is easy to see that $I_{F}=I_{f}[Y]$. For any distinct $x_{1}, x_{2} \in V\left(I_{f}\right)$ and $y \in V(Y)$, $F\left(\left(x_{1}, y\right)\right)=\left(f\left(x_{1}\right), y\right)$ and $F\left(\left(x_{2}, y\right)\right)=\left(f\left(x_{2}\right), y\right)$ hold. Since $\left.F\right|_{I_{F}}$ is an automorphism of $I_{F},\left(f\left(x_{1}\right), y\right) \neq\left(f\left(x_{2}\right), y\right)$. Hence $f\left(x_{1}\right) \neq f\left(x_{2}\right)$ and so $\left.f\right|_{I_{f}}$ is an automorphism of $I_{f}$.

Let $g \in \operatorname{End}(Y)$. Define a mapping $G$ from $V(X[Y])$ to itself by

$$
G((x, y))=(x, g(y)) \quad \forall(x, y) \in V(X[Y]) .
$$

Then $G \in \operatorname{End}(X[Y])$. Since $X[Y]$ is End-completely-regular, by Lemma $2,\left.G\right|_{I_{G}}$ is an automorphism of $I_{G}$. It is easy to see that $I_{G}=X\left[I_{g}\right]$. For any $x \in V(X)$ and $y_{1}, y_{2} \in V\left(I_{g}\right)$, $G\left(\left(x, y_{1}\right)\right)=\left(x, g\left(y_{1}\right)\right)$ and $G\left(\left(x, y_{2}\right)\right)=\left(x, g\left(y_{2}\right)\right)$. Since $\left.G\right|_{I_{G}}$ is an automorphism of $I_{G},\left(x, g\left(y_{1}\right)\right) \neq\left(x, g\left(y_{2}\right)\right)$, we get that $g\left(y_{1}\right) \neq g\left(y_{2}\right)$ and so $\left.g\right|_{I_{g}}$ is an automorphism of $I_{g}$, as required.

The following example shows that $X$ and $Y$ being End-completely-regular does not yield that $X[Y]$ is Endcompletely-regular.

Example 9. Let $X$ and $Y$ be two graphs with $V(X)=\left\{x_{1}, x_{2}\right\}$, $V(Y)=\left\{y_{1}, y_{2}\right\}, E(X)=\left\{\left\{x_{1}, x_{2}\right\}\right\}$, and $E(Y)=\phi$. By Lemma 3, $X$ and $Y$ are End-completely-regular. It is easy to see that $X[Y] \cong C_{4}$. Also by Lemma 3, this is not Endcompletely-regular.

In the following, we give some sufficient conditions for $X[Y]$ to be End-completely-regular. To this aim, we need the following result due to Fan [17].

Lemma 10 (see [17]). Let $X$ and $Y$ be two $K_{3}$-free connected graphs. If girth $(X)$ or girth $(Y)$ is odd, then End $(X[Y])=$ $\operatorname{End}(X)[\operatorname{End}(Y)]$, where End $(X)[\operatorname{End}(Y)]$ is the wreath product of the monoids End $(X)$ and End $(Y)$.

Let $X$ and $Y$ be two $K_{3}$-free connected graphs such that $\operatorname{girth}(X)$ or $\operatorname{girth}(Y)$ is odd. In [2], Fan proved that if both of $X$ and $Y$ are End-regular and one of them is unretractive, then $X[Y]$ is End-regular. Here we prove that if $X$ is an Endcompletely-regular graph and $Y$ is an unretractive graph, then $X[Y]$ is End-completely-regular. 
Theorem 11. Let $X$ and $Y$ be two $K_{3}$-free connected graphs with girth $(X)$ or girth $(Y)$ being odd, and assume that

(1) $X$ is End-completely-regular,

(2) $Y$ is unretractive.

\section{Then $X[Y]$ is End-completely-regular.}

Proof. Let $X$ and $Y$ be two graphs satisfying the assumptions. To show that $X[Y]$ is End-completely-regular, we prove that for any $F \in \operatorname{End}(X[Y])$, there exists an idempotent endomorphism $G \in \operatorname{End}(X[Y])$ such that $\rho_{F}=\rho_{G}$ and $I_{F}=I_{G}$.

Let $F \in \operatorname{End}(X)[\operatorname{End}(Y)]$. Since $\operatorname{End}(X[Y])=$ $\operatorname{End}(X)[\operatorname{End}(Y)], F=(s, f)$ for some $s \in \operatorname{End}(X)$ and $f \in \operatorname{End}(Y)^{V(X)}$. Thus, for any $u \in V(X)$, there exists $f_{u}=$ $f(u) \in \operatorname{End}(Y)$. Let $X$ and $Y$ be $K_{3}$-free connected graphs with girth $(X)$ or girth $(Y)$ being odd. By Lemma 7, for any $u \in V(X), F\left(Y_{u}\right) \subseteq Y_{v}$ for some $v \in V(X)$. Note that $Y$ is unretractive. Then $F\left(Y_{u}\right)=Y_{v}$. Since $X$ is End-completelyregular and $s \in \operatorname{End}(X)$, by Lemma 5 , there exists $t \in \operatorname{Idpt}(X)$ such that $\rho_{t}=\rho_{s}$ and $I_{t}=I_{s}$. Clearly, $I_{s}$ is an induced subgraph of $X$. Hence $I_{F}=I_{s}[Y]$ is an induced subgraph of $X[Y]$.

Since $X$ is End-completely-regular, $\left.s\right|_{I_{s}}$ is an automorphism of $I_{s}$. Thus for any $u \in I_{s}$, there exists only one vertex $u_{1} \in I_{s}$ such that $s\left(u_{1}\right)=u$. Then $F\left(Y_{u_{1}}\right)=Y_{u}$. Now for any $(u, v) \in I_{F}$, there exists only one vertex $\left(u_{1}, v_{1}\right) \in I_{F}$ such that $F\left(\left(u_{1}, v_{1}\right)\right)=(u, v)$. Define a mapping $G$ from $V(X[Y])$ to itself in the following way. If $(x, y) \in V\left(I_{F}\right)$, then $G((x, y))=$ $(x, y)$; if $(x, y) \notin V\left(I_{F}\right)$, then $F((x, y))=(u, v)$ for some $(u, v) \in V\left(I_{F}\right)$. Now let $G((x, y))=\left(u_{1}, v_{1}\right)$, where $\left(u_{1}, v_{1}\right)$ is the only vertex in $V\left(I_{F}\right)$ such that $F\left(\left(u_{1}, v_{1}\right)\right)=(u, v)$. Then it is easy to see that $G$ is well-defined. Let $(x, y) \in V(X[Y])$. If $(x, y) \in V\left(I_{F}\right)$, then $x \in I_{s}$. Thus $t(x)=x$. Hence $G((x, y))=$ $(x, y) \in Y_{t(x)}$. If $(x, y) \notin V\left(I_{F}\right)$, then $t(x)=u_{1}$. Hence $G((x, y))=\left(u_{1}, v_{1}\right) \in Y_{t(x)}$. Therefore, $G((x, y)) \in Y_{t(x)}$ for any $(x, y) \in V(X[Y])$.

Let $\left(x_{1}, y_{1}\right),\left(x_{2}, y_{2}\right) \in V(X[Y])$ be such that $\left\{\left(x_{1}, y_{1}\right),\left(x_{2}\right.\right.$, $\left.\left.y_{2}\right)\right\} \in E(X[Y])$. If $\left(x_{1}, y_{1}\right),\left(x_{2}, y_{2}\right) \in V\left(I_{F}\right)$, then $\left\{G\left(\left(x_{1}\right.\right.\right.$, $\left.\left.\left.y_{1}\right)\right), G\left(\left(x_{2}, y_{2}\right)\right)\right\}=\left\{\left(x_{1}, y_{1}\right),\left(x_{2}, y_{2}\right)\right\} \in E(X[Y])$. If $\left(x_{1}\right.$, $\left.y_{1}\right) \in V\left(I_{F}\right)$ and $\left(x_{2}, y_{2}\right) \notin V\left(I_{F}\right)$, then $x_{1} \neq x_{2}$ and $\left\{x_{1}, x_{2}\right\} \in$ $E(X)$. Thus $G\left(\left(x_{1}, y_{1}\right)\right) \in Y_{t\left(x_{1}\right)}$ and $G\left(\left(x_{2}, y_{2}\right)\right) \in Y_{t\left(x_{2}\right)}$. Since $t \in \operatorname{Idpt}(X)$ and $\left\{x_{1}, x_{2}\right\} \in E(X),\left\{t\left(x_{1}\right), t\left(x_{2}\right)\right\} \in E(X)$. Hence $\left\{G\left(\left(x_{1}, y_{1}\right)\right), G\left(\left(x_{2}, y_{2}\right)\right)\right\} \in E(X[Y])$. If $\left(x_{1}, y_{1}\right) \notin V\left(I_{F}\right)$ and $\left(x_{2}, y_{2}\right) \notin V\left(I_{F}\right)$, there are two cases.

Case 1. Assume that $\left\{x_{1}, x_{2}\right\} \in E(X)$. Then $G\left(\left(x_{1}, y_{1}\right)\right) \in$ $Y_{t\left(x_{1}\right)}$ and $G\left(\left(x_{2}, y_{2}\right)\right) \in Y_{t\left(x_{2}\right)}$. Since $t \in \operatorname{Idpt}(X)$ and $\left\{x_{1}, x_{2}\right\} \in E(X),\left\{t\left(x_{1}\right), t\left(x_{2}\right)\right\} \in E(X)$. Hence we have $\left\{G\left(\left(x_{1}, y_{1}\right)\right), G\left(\left(x_{2}, y_{2}\right)\right)\right\} \in E(X[Y])$.

Case 2. Assume that $x_{1}=x_{2}$ and $\left\{y_{1}, y_{2}\right\} \in E(Y)$. Then we have $\left(x_{1}, y_{1}\right),\left(x_{2}, y_{2}\right) \in Y_{x_{1}}$ and $G\left(\left(x_{1}, y_{1}\right)\right), G\left(\left(x_{2}, y_{2}\right)\right) \in$ $Y_{t\left(x_{1}\right)}$. Since $\left.G\right|_{Y_{x_{1}}}$ is an isomorphism from $Y_{x_{1}}$ to $Y_{t\left(x_{1}\right)}$, $\left\{G\left(\left(x_{1}, y_{1}\right)\right), G\left(\left(x_{2}, y_{2}\right)\right)\right\} \in E(X[Y])$. Therefore, $G \in$ $\operatorname{End}(X[Y])$.
If $(x, y) \in V\left(I_{F}\right)$, then $G^{2}((x, y))=G((x, y))=(x, y)$. If $(x, y) \notin V\left(I_{F}\right)$, then $G((x, y)) \in V\left(I_{F}\right)$. Thus $G^{2}((x, y))=$ $G(G((x, y)))=G((x, y))$. Hence $G \in \operatorname{Idpt}(X[Y])$. Clearly, $I_{G}=I_{F}$.

Suppose $\left[\left(x_{1}, y_{1}\right)\right]_{\rho_{F}}=\left\{\left(x_{1}, y_{1}\right),\left(x_{2}, y_{2}\right), \ldots,\left(x_{k}, y_{k}\right)\right\}$ for some $\left(x_{1}, y_{1}\right),\left(x_{2}, y_{2}\right), \ldots,\left(x_{k}, y_{k}\right) \in V(X[Y])$. In fact, it is easy to prove that $\rho_{F} \subseteq \rho_{G} \subseteq \rho_{F}$. Let $\left(x_{1}, y_{1}\right) \rho_{F}\left(x_{2}, y_{2}\right)$. Then, by the definition of $G$, we have $G\left(\left(x_{1}, y_{1}\right)\right)=G\left(\left(x_{2}, y_{2}\right)\right)=$ $\left(u_{1}, v_{1}\right)$ for some $\left(u_{1}, v_{1}\right) \in V\left(I_{F}\right)$ with $F\left(\left(x_{1}, y_{1}\right)\right)=$ $F\left(u_{1}, v_{1}\right)=F\left(\left(x_{2}, y_{2}\right)\right)$. So $\left(x_{1}, y_{1}\right) \rho_{G}\left(x_{2}, y_{2}\right)$ and thus $\rho_{F} \subseteq$ $\rho_{G} \subseteq \rho_{F}$. Hence $\rho_{F}=\rho_{G}$.

Next we start to seek the conditions for bipartite graphs $X$ and $Y$ under which $X[Y]$ is End-completely-regular.

Lemma 12. Let $X$ be a graph and $R$ be a retract of $X$. If $R[Y]$ is not End-completely-regular, then $X[Y]$ is not End-completelyregular.

Proof. Let $R$ be a retract of $X$. Then there exists $f \in \operatorname{Idpt}(X)$ such that $I_{f}=R$. Let $g \in \operatorname{End}(R[Y])$. Since $R[Y]$ is not End-completely-regular, there exists $g \in \operatorname{End}(R[Y])$ such that $g$ is not completely regular. By Lemma $2,\left.g\right|_{I_{g}}$ is not an automorphism of $I_{g}$. Thus there exist $x_{1}, x_{2} \in I_{g}$ with $x_{1} \neq x_{2}$ such that $g\left(x_{1}\right)=g\left(x_{2}\right)$. Define a mapping $F$ from $V(X[Y])$ to itself by

$$
F((x, y))=(f(x), y) \quad \forall(x, y) \in V(X[Y]) .
$$

Then $F \in \operatorname{End}(X[Y])$ and $I_{F}=R[Y]$. Now it is easy to see that $g F \in \operatorname{End}(X[Y])$ and $I_{g F}=I_{g}$. It follows from $(g F)\left(x_{1}\right)=$ $(g F)\left(x_{2}\right)$ that $\left.(g F)\right|_{I_{g F}}$ is not an automorphism of $I_{g F}$. Hence $X[Y]$ is not End-completely-regular.

Lemma 13. Let $X$ and $Y$ be two graphs. If at least one of $X$ and $Y$ is not End-completely-regular, then $X \cup Y$ is not Endcompletely-regular (where $X \cup Y$ is the disjoint union of $X$ and $Y)$.

Proof. Without loss of generality, we may suppose that $X$ is not End-completely-regular. By Lemma 2, there exists $f \in$ $\operatorname{End}(X)$ such that $\left.f\right|_{I_{f}}$ is not an automorphism of $I_{f}$. Define a mapping $F$ from $V(X \cup Y)$ to itself by

$$
F(x)= \begin{cases}f(x), & x \in V(X), \\ x, & x \in V(Y) .\end{cases}
$$

Then $F \in \operatorname{End}(X \cup Y)$. Now it is easy to see that $I_{F}=I_{f} \cup Y$ and $F(x)=f(x)$ for any $x \in V(X)$. Since $\left.f\right|_{I_{f}}$ is not an automorphism of $I_{f},\left.F\right|_{I_{F}}$ is not an automorphism of $I_{F}$. Hence $X \cup Y$ is not End-completely-regular.

Theorem 14. Let $X$ and $Y$ be two bipartite graphs. Then $X[Y]$ is End-completely-regular if and only if

(1) $X=K_{1}$ and $Y$ is End-completely-regular or

(2) $X$ is End-completely-regular and $Y=K_{1}$ or $K_{2}$. 


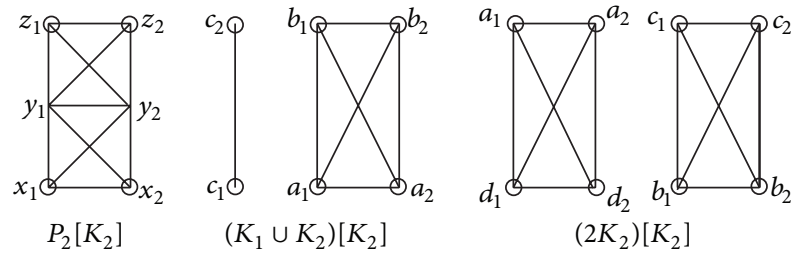

Figure 1: Graphs $P_{2}\left[K_{2}\right],\left(K_{1} \cup K_{2}\right)\left[K_{2}\right]$, and $\left(2 K_{2}\right)\left[K_{2}\right]$.

Proof

Sufficiency. Since $K_{1}[Y]=Y$ and $X\left[K_{1}\right]=X$, we have immediately that $K_{1}[Y]\left(X\left[K_{1}\right]\right)$ is End-completely-regular if and only if $Y(X)$ is End-completely-regular. If $X=Y=K_{2}$, then $X[Y]=K_{4}$. Thus $\operatorname{End}(X[Y])$ is a group. Since any group is a completely regular semigroup, $X[Y]$ is End-completelyregular. If $X=2 K_{1}$ and $Y=K_{2}$, then $X[Y]=2 K_{2}$. By Lemma 3, it is End-completely-regular. In the following, we show that $X[Y]$ is End-completely-regular for the following cases (see Figure 1).

Case 1. $X=P_{2}$ and $Y=K_{2}$. Let $f \in \operatorname{End}(X[Y])$. If $\left[x_{1}\right]_{\rho_{f}}=\left\{x_{1}\right\}$, then $\left[x_{2}\right]_{\rho_{f}}=\left\{x_{2}\right\}$. Otherwise, $f\left(x_{2}\right)=$ $f\left(z_{1}\right)$ or $f\left(x_{2}\right)=f\left(z_{2}\right)$. Without loss of generality, we can suppose $f\left(x_{2}\right)=f\left(z_{1}\right)$. Since $z_{1}$ is adjacent to every vertex of $\left\{z_{2}, y_{1}, y_{2}\right\}$ and $\left\{x_{1}, x_{2}\right\} \in E(X[Y]), f\left(z_{1}\right)$ is adjacent to every vertex of $\left\{f\left(z_{2}\right), f\left(y_{1}\right), f\left(y_{2}\right), f\left(x_{1}\right)\right\}$. Note that there is no vertex in $X[Y]$ adjacent to 4 vertices. This is a contradiction. Hence $f \in \operatorname{Aut}(X[Y])$ and so $f$ is completely regular. If $\left[x_{1}\right]_{\rho_{f}} \neq\left\{x_{1}\right\}$, then $f\left(x_{1}\right)=f\left(z_{1}\right)$ or $f\left(x_{1}\right)=$ $f\left(z_{2}\right)$. Without loss of generality, we can suppose $f\left(x_{1}\right)=$ $f\left(z_{1}\right)$. Then $f\left(x_{2}\right)=f\left(z_{2}\right)$. Otherwise, a similar argument as above will show that $f\left(x_{1}\right)$ is adjacent to every vertex of $\left\{f\left(x_{2}\right), f\left(y_{1}\right), f\left(y_{2}\right), f\left(z_{2}\right)\right\}$, which yield a contradiction. Thus $I_{f} \cong K_{4}$. Since any endomorphism $f$ maps a clique to a clique of the same size, $f\left(I_{f}\right)=I_{f}$. By Lemma $2, f$ is completely regular. Hence $P_{2}\left[K_{2}\right]$ is End-completely-regular.

Case 2. $X=K_{1} \cup K_{2}$ and $Y=K_{2}$. Let $f \in$ $\operatorname{End}(X[Y])$. If $\left[c_{1}\right]_{\rho_{f}}=\left\{c_{1}\right\}$, then $\left[c_{2}\right]_{\rho_{f}}=\left\{c_{2}\right\}$. Otherwise, $f\left(c_{2}\right) \in\left\{f\left(a_{1}\right), f\left(a_{2}\right), f\left(b_{1}\right), f\left(b_{2}\right)\right\}$. Without loss of generality, we can suppose $f\left(c_{2}\right)=f\left(a_{1}\right)$. Since any endomorphism $f$ maps a clique to a clique of the same size and there is only one clique of size 4 in $X[Y]$, $\left\{f\left(a_{1}\right), f\left(a_{2}\right), f\left(b_{1}\right), f\left(b_{2}\right)\right\}=\left\{a_{1}, a_{2}, b_{1}, b_{2}\right\}$. Note that $\left\{c_{1}\right.$, $\left.c_{2}\right\} \in E(X[Y])$. Then $\left\{f\left(c_{1}\right), f\left(c_{2}\right)\right\}=\left\{f\left(c_{1}\right), f\left(a_{1}\right)\right\} \in$ $E(X[Y])$. Thus $f\left(c_{1}\right) \in\left\{f\left(a_{1}\right), f\left(a_{2}\right), f\left(b_{1}\right), f\left(b_{2}\right)\right\}$, which is a contradiction. Clearly, $[x]_{\rho_{f}}=\{x\}$ for any $x \in\left\{a_{1}, a_{2}, b_{1}, b_{2}\right\}$. Hence $f \in \operatorname{Aut}(X[Y])$ and so $f$ is completely regular. If $\left[c_{1}\right]_{\rho_{f}} \neq\left\{c_{1}\right\}$, then $f\left(c_{1}\right)=f(t)$ for some $t \in\left\{a_{1}, a_{2}, b_{1}, b_{2}\right\}$. Without loss of generality, we can suppose $f\left(c_{1}\right)=f\left(a_{1}\right)$. Then $f\left(c_{2}\right) \in\left\{f\left(a_{2}\right), f\left(b_{1}\right), f\left(b_{2}\right)\right\}$. Thus $I_{f} \cong K_{4}$. Hence $f\left(I_{f}\right)=I_{f}$ and $f$ is completely regular. Consequently, $\left(K_{1} \cup\right.$ $\left.K_{2}\right)\left[K_{2}\right]$ is End-completely-regular.

Case 3. $X=2 K_{2}$ and $Y=K_{2}$. Let $f \in \operatorname{End}(X[Y])$. If $[x]_{\rho_{f}}=\{x\}$ for any $x \in V(X[Y])$, then $f \in \operatorname{Aut}(X[Y])$ and so $f$ is completely regular. If $f(x)=f(y)$ for some $x, y \in V(X[Y])$ with $x \neq y$, without loss of generality, we can suppose $f\left(a_{1}\right)=f\left(c_{1}\right)$. Since $b_{1}, b_{2}, c_{1}, c_{2}$ is a clique of size 4 in $X[Y], f\left(b_{1}\right), f\left(b_{2}\right), f\left(c_{1}\right), f\left(c_{2}\right)$ is also a clique of size 4 in $X[Y]$. Note that $a_{2}, d_{1}, d_{2}$ are adjacent to $a_{1}$. Then $f\left(a_{2}\right), f\left(d_{1}\right), f\left(d_{2}\right)$ are adjacent to $f\left(a_{1}\right)=f\left(c_{1}\right)$. Thus $f\left(a_{2}\right), f\left(d_{1}\right), f\left(d_{2}\right) \in\left\{f\left(b_{1}\right), f\left(b_{2}\right), f\left(c_{2}\right)\right\}$ and $I_{f} \cong K_{4}$. Hence $f\left(I_{f}\right)=I_{f}$ and $f$ is completely regular. Consequently, $\left(2 K_{2}\right)\left[K_{2}\right]$ is End-completely-regular.

Necessity. We only need to show that $X[Y]$ is not Endcompletely-regular in the following cases.

Case $1\left(X=K_{2}\right)$. Then $X[Y]=Y+Y$. By Lemma $4, K_{2}[Y]$ is not End-completely-regular for the corresponding $Y$.

Case $2\left(X=P_{2}\right)$. Then $K_{2}$ is a retract of $X$. Since $K_{2}[Y]$ is not End-completely-regular for $Y=P_{2}, 2 K_{1}, 2 K_{2}, K_{1} \cup K_{2}$, by Lemma $12, P_{2}[Y]$ is not End-completely-regular for the corresponding $Y$.

Case $3\left(X=2 K_{1}\right)$. Then $X[Y]=2 Y$. If $Y$ is bipartite, then $X[Y]$ is also bipartite. By Lemma $3,\left(2 K_{1}\right)[Y]$ is not Endcompletely-regular for the corresponding $Y$.

Case $4\left(X=2 K_{2}\right)$. Then $X[Y]=2(Y+Y)$. Since $Y+Y$ is not End-completely-regular for $Y=P_{2}, 2 K_{1}, 2 K_{2}, K_{1} \cup K_{2}$, by Lemma $13,\left(2 K_{2}\right)[Y]$ is not End-completely-regular for the corresponding $Y$.

Case $5\left(X=K_{1} \cup K_{2}\right)$. Then $X[Y]=Y \cup(Y+Y)$. Since $Y+Y$ is not End-completely-regular for $Y=P_{2}, 2 K_{1}, 2 K_{2}, K_{1} \cup K_{2}$, by Lemma $13,\left(K_{1} \cup K_{2}\right)[Y]$ is not End-completely-regular for the corresponding $Y$.

Next we start to seek the conditions for a lexicographic product of bipartite graphs $X$ and $Y$ under which $X[Y]$ is End-inverse.

Theorem 15. Let $X$ and $Y$ be two graphs. If $X[Y]$ is Endinverse, then both $X$ and $Y$ are End-inverse.

Proof. Since $X[Y]$ is End-inverse, $X[Y]$ is End-regular. By Lemma 1, both $X$ and $Y$ are End-regular. To show that $X$ is End-inverse, we only need to prove that the idempotents of $\operatorname{End}(X)$ commute.

Let $f_{1}$ and $f_{2}$ be two idempotents in $\operatorname{End}(X)$. Define two mappings $g_{1}$ and $g_{2}$ from $V(X[Y])$ to itself by

$$
\begin{array}{ll}
g_{1}((x, y))=\left(f_{1}(x), y\right) & \forall(x, y) \in V(X[Y]), \\
g_{2}((x, y))=\left(f_{2}(x), y\right) & \forall(x, y) \in V(X[Y]) .
\end{array}
$$

Then $g_{1}$ and $g_{2}$ are two idempotents of $\operatorname{End}(X[Y])$ and so $g_{1} g_{2}=g_{2} g_{1}$, since $X[Y]$ is End-inverse. For any $(x, y) \in$ $V(X[Y])$, we have

$$
\begin{aligned}
\left(g_{1} g_{2}\right)((x, y)) & =g_{1}\left(\left(f_{2}(x), y\right)\right)=\left(\left(f_{1} f_{2}\right)(x), y\right) \\
& =\left(g_{2} g_{1}\right)((x, y))=\left(\left(f_{2} f_{1}\right)(x), y\right) .
\end{aligned}
$$

Clearly, $f_{1} f_{2}=f_{2} f_{1}$. Hence $X$ is End-inverse. 
Similarly, let $f_{3}$ and $f_{4}$ be two idempotents in $\operatorname{End}(Y)$. Define two mappings $g_{3}$ and $g_{4}$ from $V(X[Y])$ to itself by

$$
\begin{array}{ll}
g_{3}((x, y))=\left(x, f_{3}(y)\right) & \forall(x, y) \in V(X[Y]), \\
g_{4}((x, y))=\left(x, f_{4}(y)\right) & \forall(x, y) \in V(X[Y]) .
\end{array}
$$

Then $g_{3}$ and $g_{4}$ are two idempotents of $\operatorname{End}(X[Y])$ and so $g_{3} g_{4}=g_{4} g_{3}$, since $X[Y]$ is End-inverse. For any $(x, y) \in$ $V(X[Y])$, we have

$$
\begin{aligned}
\left(g_{3} g_{4}\right)((x, y)) & =\left(x,\left(f_{3} f_{4}\right)(y)\right)=\left(g_{4} g_{3}\right)((x, y)) \\
& =\left(x,\left(f_{4} f_{3}\right)(y)\right) .
\end{aligned}
$$

Clearly, $f_{3} f_{4}=f_{4} f_{3}$. Hence $Y$ is End-inverse, as required.

The next theorem characterizes the End-inverse lexicographic products of bipartite graphs.

Theorem 16. Let $X$ and $Y$ be two bipartite graphs. Then $X[Y]$ is End-inverse if and only if $X[Y]$ is one of $K_{1}\left[K_{1}\right], K_{1}\left[K_{2}\right]$, $K_{2}\left[K_{1}\right]$, and $K_{2}\left[K_{2}\right]$.

Proof

Necessity. This follows directly from Lemma 6 and Theorem 15.

Sufficiency. It is easy to see that $K_{1}\left[K_{1}\right]=K_{1}, K_{1}\left[K_{2}\right]=$ $K_{2}\left[K_{1}\right]=K_{2}$, and $K_{2}\left[K_{2}\right]=K_{4}$ are End-inverse, since they are unretractive.

\section{Conflict of Interests}

The authors declare that there is no conflict of interests regarding the publication of this paper.

\section{Acknowledgments}

The authors want to express their gratitude to the referees for their helpful suggestions and comments. This research was partially supported by the National Natural Science Foundation of China (nos. 11301151 and 11226047), the Key Project of the Education Department of Henan Province (no. 13A110249), and the Project of Science and Technology Department of Henan Province (no. 132300410411).

\section{References}

[1] E. Wilkeit, "Graphs with a regular endomorphism monoid," Archiv der Mathematik, vol. 66, no. 4, pp. 344-352, 1996.

[2] S. Fan, "On end-regular graphs," Discrete Mathematics, vol. 159, no. 1-3, pp. 95-102, 1996.

[3] H. Hou and Y. Luo, "Graphs whose endomorphism monoids are regular," Discrete Mathematics, vol. 308, no. 17, pp. 3888-3896, 2008.

[4] H. Hou, R. Gu, and X. Li, "End-completely-regular and endinverse joins of graphs," Ars Combinatoria. In press.
[5] H. Hou, Y. Luo, and X. Fan, "End-regular and end-orthodox joins of split graphs," Ars Combinatoria, vol. 105, pp. 305-318, 2012.

[6] H. L. Hou, Y. F. Luo, and R. Gu, "The join of split graphs whose half-strong endomorphisms form a monoid," Acta Mathematica Sinica, vol. 26, no. 6, pp. 1139-1148, 2010.

[7] W. Li and J. Chen, "Endomorphism—regularity of split graphs," European Journal of Combinatorics, vol. 22, no. 2, pp. 207-216, 2001.

[8] W. Li, "Graphs with regular monoids," Discrete Mathematics, vol. 265, no. 1-3, pp. 105-118, 2003.

[9] M. Böttcher and U. Knauer, "Endomorphism spectra of graphs," Discrete Mathematics, vol. 109, no. 1-3, pp. 45-57, 1992.

[10] J. M. Howie, Fundamentals of Semigroup Theory, Clarendon Press, Oxford, UK, 1995.

[11] A. V. Kelarev, J. Ryan, and J. Yearwood, "Cayley graphs as classifiers for data mining: the influence of asymmetries," Discrete Mathematics, vol. 309, no. 17, pp. 5360-5369, 2009.

[12] A. V. Kelarev, Graph Algebras and Automata, Marcel Dekker, New York, NY, USA, 2003.

[13] U. Knauer, Algebraic Graph Theory: Morphisms, Monoids and Matrices, Walter de Gruyter, Berlin, Germany, 2011.

[14] P. Petrich and N. R. Reilly, Completely Regular Semigroups, John Wiley \& Sons, New York, NY, USA, 1999.

[15] S. Fan, "End-regular graphs," Journal of Jinan University, vol. 18, pp. 1-7, 1997.

[16] W. Li, "Split graphs with completely regular endomorphism monoids," Journal of Mathematical Research and Exposition, vol. 26, no. 2, pp. 253-263, 2006.

[17] S. H. Fan, "The endomorphism monoid of the lexicographic product of two graphs," Acta Mathematica Sinica, vol. 38, no. 2, pp. 248-252, 1995. 


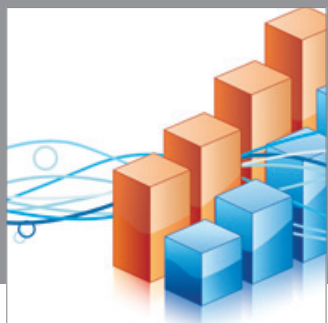

Advances in

Operations Research

mansans

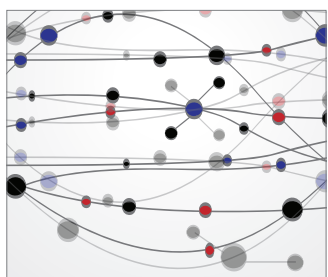

The Scientific World Journal
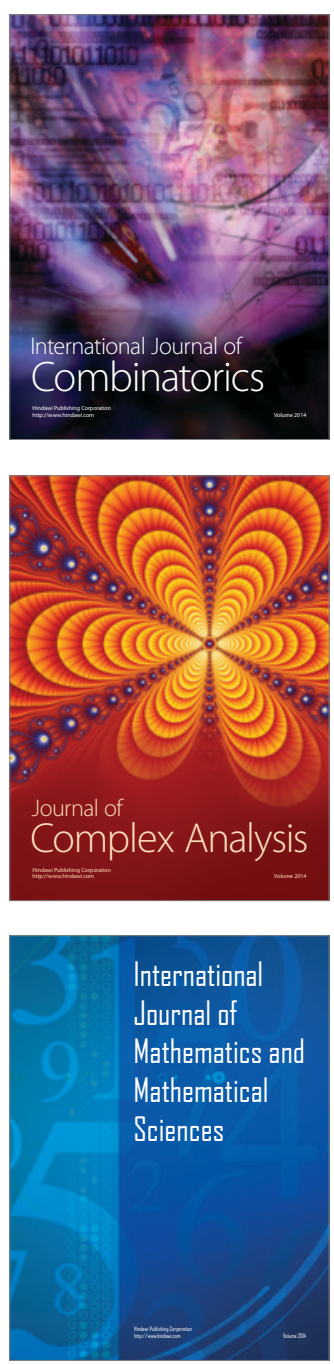
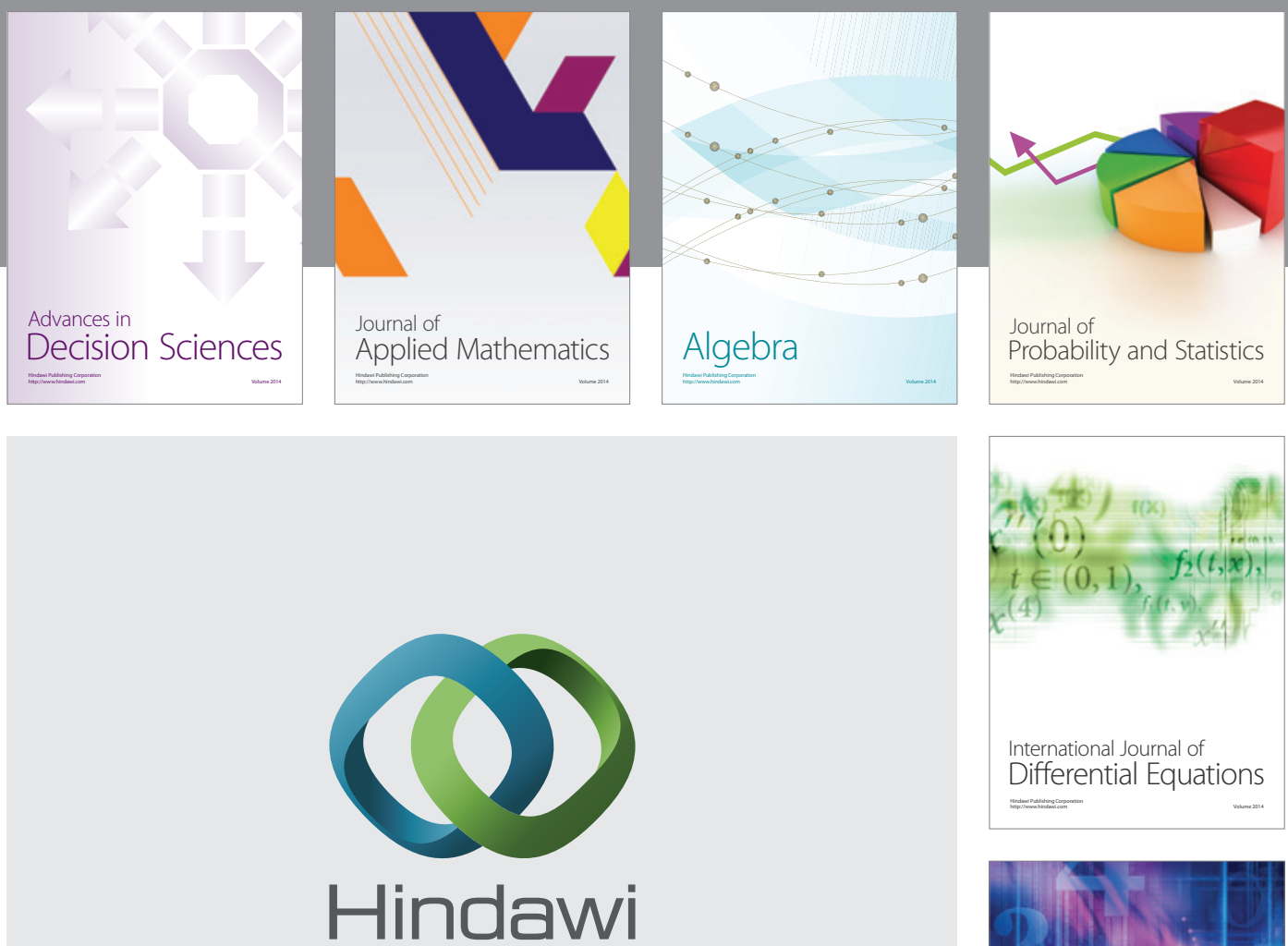

Submit your manuscripts at http://www.hindawi.com
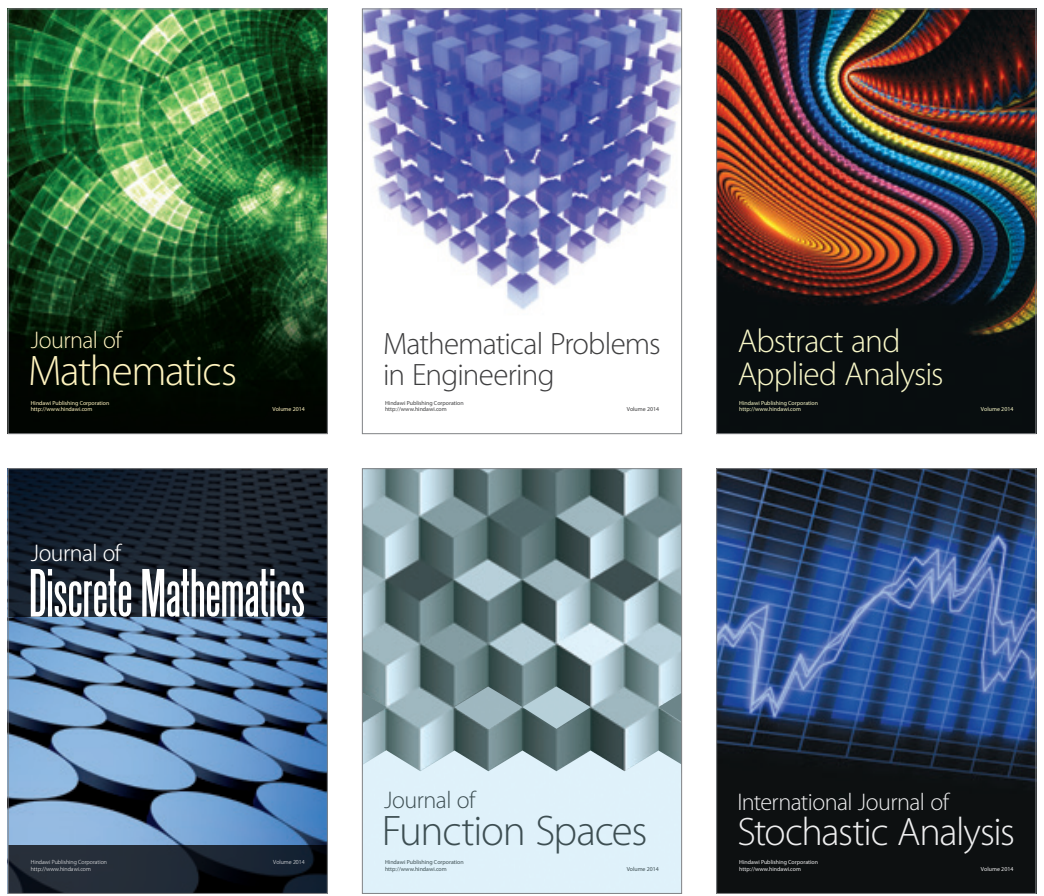

Journal of

Function Spaces

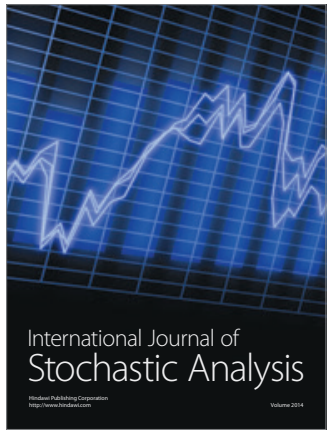

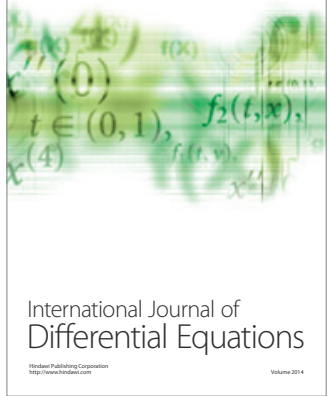
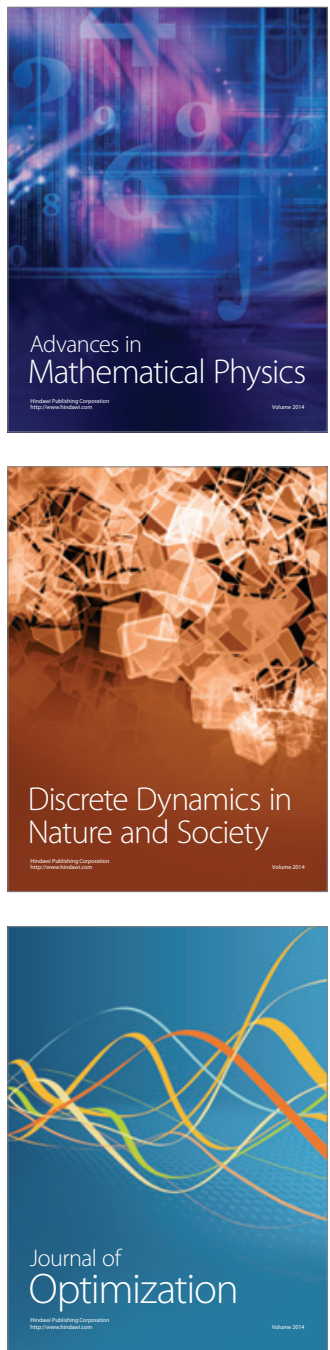